\title{
CORRELATION BETWEEN VERTICAL GROWTH PATTERNS OF THE JAWS AND SELLA TURCICA'S LINEAR DIMENSIONS USING CONE BEAM COMPUTED TOMOGRAPHY IN AN EGYPTAIN SUBPOPULATION
}

\author{
Asmaa Youssry Abdallah*
}

\begin{abstract}
The sella turcica is one of the most important landmarks used by orthodontics in analysis of growth and treatment planning. Therefore, the purpose of this retrospective study was to detect whether there is a correlation between the sella turcica's linear dimensions and the vertical growth patterns of the jaws using CBCT in an Egyptian subpopulation. 144 CBCT scans of adult patients (68 males and 76 females) were taken from the archive of the Oral Radiology Department, Faculty of Dentistry, Suez Canal University. CBCT images were divided according to the vertical growth pattern of both maxilla and mandible into three groups; hypodivergent, normodivergent and hyperdivergent. The sella turcica diameter, length and depth were measured individually for each case. There were no statistically significant differences found between sella turcica's length and diameter measurements and vertical growth pattern of the jaws. While significant difference was found regarding the depth only between the three groups where the p-value was found to be 0.001 . Conclusion: CBCT could be used as a highly accurate radiographic modality in assessing the linear dimensions of sella turcica. The sellar depth was the only linear measurement found to be affected by the vertical growth pattern of the jaws.
\end{abstract}

\section{INTRODUCTION}

In the craniofacial region, several reference points are used for orthodontic treatment assessment and lateral cephalograms tracing. One of the most important landmarks is the sella ( $\mathrm{S}$ ) point, which is of special significance due to its role as a reference point in the assessment of intermaxillary relations and cranial morphology. Sella point is situated in the center of the sella turcica, with the turcica enclosing the pituitary gland in the base of the cranium. ${ }^{(1)}$

Sella turcica has a complex anatomy; it is enclosed in the sphenoid bone which has a central cavity possessing two lesser and two greater wings in addition to two pterygoid processes. The central cavity of the sella turcica encloses the pituitary gland. The tuberculum sella forms the anterior

\footnotetext{
* Lecturer of Oral Radiology, Faculty of Dentistry, Suez Canal University
} 
border and an anterior elevation on the body of the sphenoid bone, while posteriorly, the dorsum sella is situated and is formed by the square bony plate of the body of the sphenoid bone. ${ }^{(1)}$

Any abnormalities or pathosis in the pituitary gland result in changes in the size and shape of the sella turcica which can be manifested clinically as disturbances in the glandular hormones regulation and secretion such as prolactin growth hormones, TSH and follicular stimulating hormone. (2,3) These disturbances will in return lead to growth abnormalities such as gigantism, acromegaly, intrasellar pituitary primary tumors or syndromes like Sheehan's or Williams syndrome. ${ }^{(4,5)}$

Several previous studies conducted on the shape of sella turcica concluded that sella turcica morphology is established in the early embryonic life. A study was performed by Kjaer et al. on 16 children born with myelomeningocele, where he studied the profile radiographs of them and found an altered shape of sella present during their foetal life. ${ }^{(6)}$ Furthermore, an evident change in the sella turcica's shape was found by Russell and Kjaer among prenatal and postnatal children with fragile $\mathrm{X}$ and Down syndrome. ${ }^{(7)}$

Different studies were conducted using conventional lateral cephalograms by Valizadeh et $\mathrm{al}^{(8)}$, Alkofide ${ }^{(9)}$ and Motawani et al ${ }^{(10)}$ trying to correlate between the shape and size of the sella turcica and the anteroposterior relationship of the jaws within different populations. In addition to other multiple studies performed assessing the vertical growth pattern of the jaws and its relation to the shape and size of the sella turcica. ${ }^{(8-10)}$

In a study performed by Rohria and Jain ${ }^{(1)}, 90$ patients were divided equally into three groups, a hypodivergent, normodivergent and hyperdivergent ones according to their vertical cepahalometric readings. Furthermore, they studied the sella's shape as well regarding, its dimensions in terms of diameter, length and depth and correlated those findings together. A significant correlation was found between the vertical growth pattern and sellar shape and morphology. ${ }^{(11)}$

Similarly, Konwar et al ${ }^{(12)}$ studied a sample of 100 Indian patients that were divided into a high and low angle groups according to their mandibular growth pattern. In relation to their morphological sellar dimensional differences regarding diameter, length and depth, no statistically significant differences were found in linear dimensions between the two compared groups except for the sellar length, which was significantly larger in the low angled patients than the high angled ones. ${ }^{(12)}$

On the other hand, Yasa et al ${ }^{(13)}$ found no significant difference in the length of sella turcica among the different vertical growth pattern groups that they studied. However, they found that the sellar diameter was insignificantly greater in the high angle and low angle individuals than the average angle ones. Increased sellar depth was also associated with patients having high angle skeletal structures. $^{(13)}$

From the above mentioned review, it could be noted that although several previous studies were correlating the morphology of the sella turcica to different vertical growth patterns using lateral cephalograms and none to our knowledge was performed on an Egyptian sample. Therefore, the aim of the present study was to evaluate whether there is a correlation between the sella turcica's linear dimensions and vertical growth pattern of the jaw using CBCT in an Egyptian subpopulation.

\section{MATERIALS AND METHODS}

The present retrospective study was performed on 144 CBCT records obtained from the archives of the Oral Radiology Department, Faculty of Dentistry, Suez Canal University. Out of respect for patient confidentiality, all personal information concerning the patients other than age was hidden. The full skull CBCT images were collected during 
the period of January 2017 to July 2019. The scans were for adult patients ( 68 males and 76 females) ranging in age between 20 and 40 years.

The CBCT scans were selected according to the following criteria:

1. High quality full skull CBCT images that were free from artifacts caused by metallic objects that may impair clear visuality of the sella turcica.

2. The CBCT images were for subjects free from any craniofacial abnormalities.

3. The CBCT images revealed no apparent pathology or facial asymmetry in the maxillofacial region.

\section{Cone-beam computed tomography scanning protocol}

All CBCT scans assessed in the present study were scanned using the SCANORA 3DX scanner. (Scanora 3DX, Soredex, Finland). The field of view was fixed at 240x165mm for all images using standard resolution mode. The operating parameters were $90 \mathrm{KVp}, 10 \mathrm{~mA}$ and the scan time was about 6 seconds. The voxel size was $0.5 \mathrm{~mm}$ using flat panel a-Si detector. The acquired data was transferred into DICOM format, then exported into On Demand 3D application software (On Demand Cybermed. Co., Seoul, Korea) for image analysis and measurements.

\section{Grouping of CBCT images}

From the Lateral view of the 3D CBCT volume, images were divided according to the growth pattern into 3 groups : normal, horizontal, and vertical where images have to fulfill all the following angles:

$\leftarrow$ Based on the gonial angle: where it was considered normal between $119^{\circ}$ and $129^{\circ}$, while horizontal growth pattern if less than $119^{\circ}$ and vertical growth pattern if more than $129^{\circ}$. Fig $3 \mathrm{~B}$

$\leftarrow$ The angle between the lower border of the mandible and palatal plane $\mathrm{Mn} / \mathrm{PL}$ was considered: normal between $19^{\circ}$ and $27^{\circ}$, while horizontal growth pattern if less than $19^{\circ}$ and vertical growth pattern if more than $27^{\circ} .{ }^{(14)} \mathrm{Fig}$ $3 \mathrm{~A}$

$\leftarrow$ The angle between mandible and Frankfort plane angle $\mathrm{Mn} / \mathrm{FH}$ : was considered normal between $22^{\circ}$ and $28^{\circ}$, while horizontal growth pattern if less than $22^{\circ}$ and vertical growth pattern if more than $28^{\circ} .^{(14)}$ Fig 3B

$\leftarrow$ The angle between mandible and sella turcica and nasion plane $\mathrm{Mn} / \mathrm{SN}$ : was considered normal between $27^{\circ}-37^{\circ}$, while horizontal growth pattern if less than $27^{\circ}$ and vertical growth pattern if more than $37^{\circ} .^{(14)}$ Fig $3 \mathrm{~A}$

The linear measurements of length, depth, and diameter of the sella turcica were obtained using the method described by Silverman as follows: ${ }^{(15)}$

The axial view at the level of the anterior nasal spine was used as a reference line for linear measurements taken from the CBCT images. Subsequently, 1-mm sagittal slices, were made distal to the right side of the midsagittal plane so as to identify the level of sella turcica, for the measurement of the length, depth and diameter of the sella turcica. ${ }^{(16)}$ (Fig. 1 and 2) as follows:

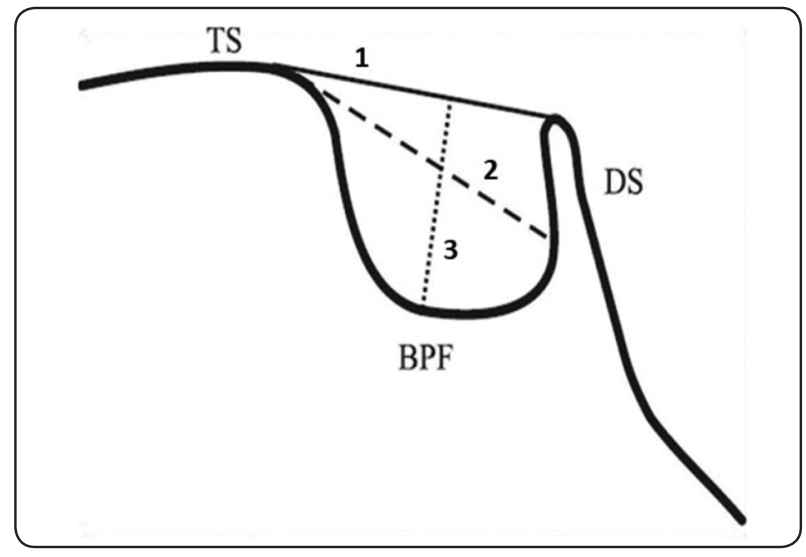

Fig. (1) The sella turcica length (1), diameter (2) and depth (3); TS - tuberculum sella; DS - dorsum sella; BPF base of pituitary fossa (15). 
$\leftarrow$ Length of the sella turcica is defined as the distance between the tip of tuberculum sellae and dorsum sellae. ${ }^{(15)}$

$\leftarrow$ Depth is the deepest point of the floor of sella turcica. ${ }^{(15)}$

$\leftarrow$ Diameter is the furthest point on the posteriorinferior aspect of the pituitary fossa to the most superior point on the tuberculum sella. ${ }^{(15)}$

Radiologic evaluation was performed by an experienced dento-maxillofacial radiologist of 7 years' experience. The intra-observer agreement was performed by reassessing the greater part of the images twice, with a 4-week interval between both viewings.

\section{Statistical Analysis}

Numerical data were explored for normality by checking the distribution of data and using tests of normality (Kolmogorov-Smirnov and ShapiroWilk tests). Data showed normal (parametric) distribution. Data were presented as mean, standard deviation (SD) and 95\% Confidence Interval (95\% CI) values. One-way ANOVA was used to compare

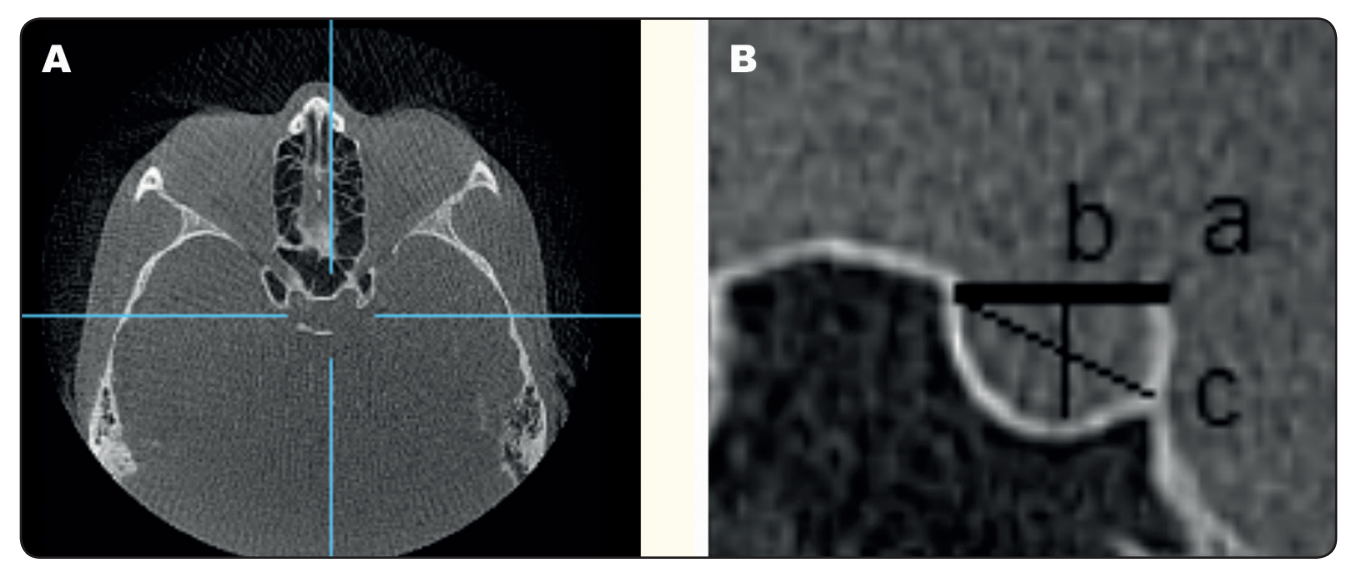

Fig. (2) i) The blue line shows the midsagittal slice selected as a reference line for the measurement of length, diameter, and depth. ii) The reference lines used for measuring the sella turcica size on the midsagittal slice: a) bold line, length of sella; b) line, perpendicular to length depth of sella; c) diameter of sella.

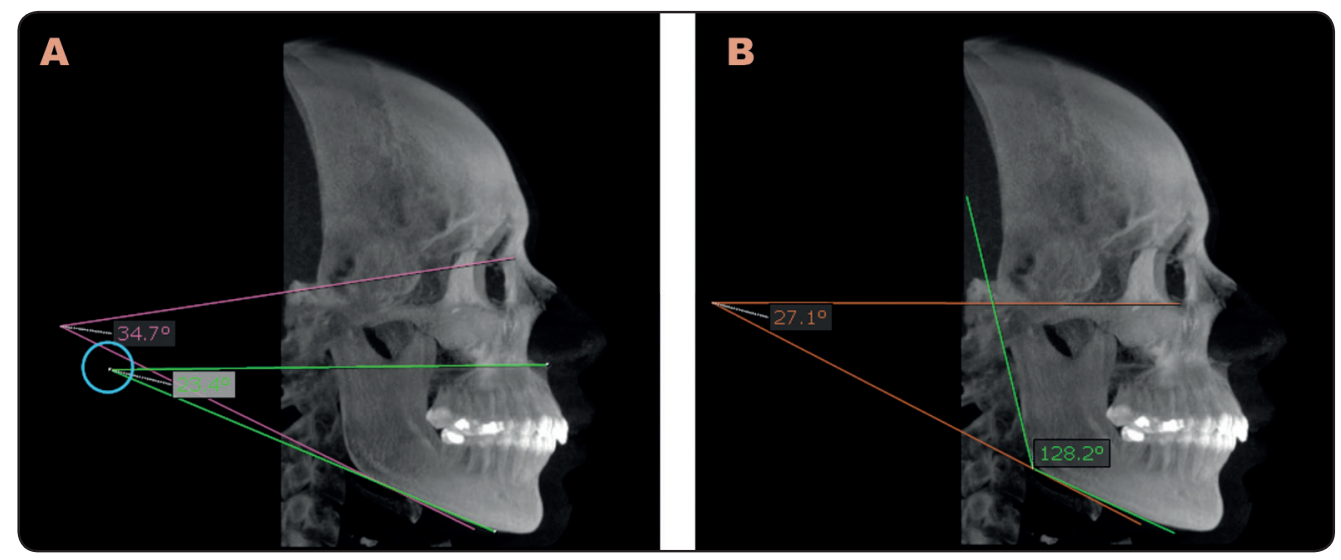

Fig. (3) Lateral view of the 3D CBCT volume (A) where the green lines shows the angle between the lower border of the mandible and palatal plane $\mathrm{Mn} / \mathrm{PL}$, the pink lines shows the angle between mandible and sella turcica and nasion plane $\mathrm{Mn} / \mathrm{SN},(\mathrm{B})$ the orange lines shows the angle between mandible and Frankfort plane angle $\mathrm{Mn} / \mathrm{FH}$ and the green lines shows the gonial angle of normal patient. 
between the three groups. Bonferroni's post-hoc test was used for pair-wise comparisons. The significance level was set at $P \leq 0.05$. Statistical analysis was performed with IBM ${ }^{\circledR}$ SPSS $^{\circledR}$ Statistics Version 20 for Windows.

\section{RESULTS}

The mean and standard deviation and the results of one-way ANOVA test for comparisons between sella turcica measurements in the three groups are shown in table (1). As regards sella turcica diameter; there was no statistically significant difference between the three groups $(P$-value $=0.307)$.

While for Sella Turcica depth; there was a statistically significant difference between the three groups $(P$-value $<0.001)$. Horizontal growth group showed the statistically highest mean depth. There was no significant difference between sella turcica depth of the other two groups; both showed significantly lower mean values.

As regards Sella Turcica length; there was no statistically significant difference between the three groups $(P$-value $=0.105)$.

\section{DISCUSSION}

The current retrospective study was conducted to explore a correlation exists between the linear measurements of sella turcica and different vertical growth pattern of the jaws using CBCT. One hundred forty-four CBCT scans of adults were examined. Since multiple previous studies stated that no differences exist between males and females regarding sella turcica size, therefore there was no sex predilection in the present study. ${ }^{(9,17)}$

The sella turcica measurements are very importance as the differences and changes in its

TABLE (1): Descriptive statistics and results of one-way ANOVA test for correlation between sella turcica measurements in the three groups

\begin{tabular}{|c|c|c|c|}
\hline & Diameter & Depth & Length \\
\hline Normal growth $(\mathrm{n}=43)$ & & & \\
\hline Mean (SD) & $11.52(1.09)$ & $7.05(1.44)^{\mathrm{B}}$ & $10.13(1.73)$ \\
\hline $95 \% \mathrm{CI}$ & $11.18-11.85$ & $6.61-7.49$ & $9.6-10.66$ \\
\hline Vertical growth ( $\mathrm{n}=73)$ & & & \\
\hline Mean (SD) & $11.67(1.27)$ & $7.26(1.44)^{\mathrm{B}}$ & $10(1.69)$ \\
\hline $95 \% \mathrm{CI}$ & $11.38-11.97$ & $6.92-7.59$ & $9.6-10.39$ \\
\hline Horizontal growth ( $\mathrm{n}=28)$ & & & \\
\hline Mean (SD) & $11.73(0.55)$ & $8.6(0.55)^{\mathrm{A}}$ & $11.03(0.6)$ \\
\hline $95 \% \mathrm{CI}$ & $11.52-11.94$ & $8.38-8.81$ & $10.8-11.26$ \\
\hline$P$-value & 0.307 & $<0.001^{*}$ & 0.105 \\
\hline Effect size (Eta squared) & 0.022 & 0.096 & 0.033 \\
\hline
\end{tabular}

*: Significant at $P \leq 0.05$, different superscripts in the same column are statistically significantly different 
shape and size could be indicative of pituitary gland disease. The normal dimensions of length, diameter and depth of sella turcica range from 4 to $16 \mathrm{~mm} .{ }^{(9,17,18)}$

In the present study the mean values for the sella measurements were in accordance with those reported in previous studies. Tetradis and Kantor ${ }^{(19)}$ reported a mean sella turcica length and depth of $10.9 \pm 1.8 \mathrm{~mm}$ and $7.6 \pm 1.7 \mathrm{~mm}$ respectively. Canigur Bavbek and Dincer ${ }^{(20)}$ found the normal length and depth values to be $10.9 \pm 1.73 \mathrm{~mm}$ and $8.29 \pm 1.66 \mathrm{~mm}$ respectively. As regards the diameter of the sella turcica the findings of the current study are in consistent with those of Soakar and Nawale ${ }^{(21)}$ who reported a mean diameter of $11.18 \pm 1.34 \mathrm{~mm}$.

Only a few studies were conducted on the correlation between the different vertical growth patterns and the sella turcica size. Those studies mainly used lateral cephalograms for their measurements. To our knowledge none was conducted using CBCT. The inherent drawback of superimposition found in lateral cephalograms is nowadays overcome when using 3D CBCTs for diagnostic purposes in the field of orthodontics.

The results of the present study revealed no significant differences in the sella dimensions between the three groups, except for the depth which was found to be higher in the horizontal growth pattern group.

Konwar et al ${ }^{(12)}$ found the sellar length was larger in the low angle group with no differences in the other sellar dimensions, which contrasts the findings of the current work, where only the depth showed a statistically significant difference.

The findings of Yasa et al ${ }^{(13)}$ were in accordance with the present findings in terms of no length differences between the study groups. On the other hand, they found the sellar depth to be increased in the vertical growth group while the current study found the increase to be present in the horizontal growth group.
Those differences in findings could be attributed to the use of lateral cephalograms in the above mentioned studies compared to the use of CBCT in the present one resulting in different magnifications. The use of three-dimensional imaging for studying the pituitary gland size was recommended by Magat and Ozcan Sener ${ }^{(22)}$.

Those differences could be also attributed to the different ethnic groups, distribution or the age groups investigated in the different studies.

The one thing found in common in all studies was the fact that a correlation exists between the sellar dimensions and the vertical growth patterns, which needs to be studied more extensively.

\section{CONCLUSIONS}

1. CBCT could be used as a highly accurate radiographic technique in assessing the linear dimensions of the sella turcica.

2. The sellar depth was the only linear measurement found to be affected by the vertical growth patterns of the jaws.

3. When examining sella turcica dimensions, the results of the present study could be used as a reference for Egyptian subjects regarding sella turcica size.

\section{REFERENCES}

1. Tekiner H, Acer N, Kelestimur F. Sella turcica: an anatomical, endocrinological, and historical perspective. Vol 18; 2014.

2. Pisaneschi M, Kapoor G. Imaging the sella and parasellar region. Neuroimaging Clin N Am. 2005; 15 (1): 203-219.

3. Elster AD. Imaging of the sella: anatomy and pathology. Semin Ultrasound CT MR. 1993; 14 (3): 182-194.

4. Elster AD. Modern imaging of the pituitary. Radiology. 1993; 187:1-14

5. Andredaki M, Koumantanou A, Dorotheou D, Halazonetis DJ. A cephalometric morphometric study of the sella turcica. Eur J Orthod. 2007; 29(5): 449-456. 
6. Kjaer I, Wagner A, Madsen P, Blichfeldt S, Rasmussen K, Russell B. The sella turcica in children with lumbosacral myelomeningocele. Eur J Orthod 1998; 20: 443-448.

7. Russell BG, Kjaer I. Postnatal structure of the sella in Down syndrome. Am J Med Gen. 1999; 19: 183-188.

8. Valizadeh S, Shahbeig S, Mohseni S, Azimi F, Bakhshandeh H. Correlation of shape and size of sella turcica with the type of facial skeletal class in an Iranian group. Iran J Radiol. 2015 Jul; 12(3): e16059.

9. Alkofide EA. The shape and size of the sella turcica in skeletal Class I, Class II, and Class III Saudi subjects. Eur J Orthod. 2007; 29(5):457-463.

10. Motwani MB, Biranjan R, Dhole A, Choudhary AB, Mohite A. A study to evaluate the shape and size of sella turcica and its correlation with the type of malocclusion on lateral cephalometric radiographs. IOSR-JDMS 2017; 16(6): 126-132.

11. Rohria R, Jain S. Sellar dimension in skeletal class II subjects with different growth patterns. Journal of Pierre Fauchard Academy (India Section). 2015;29(1):26-31.

12. Konwar SK, Singhla A, Bayan R. Morphological (Length, Depth, and Diameter) Study of Sella Turcica in Different Mandibular Growth Patterns in Indians. International Journal of Dental and Medical Speciality. 2016;3(3):4.

13. Yasa Y, Büyük SK, Benkli YA, Arslan A, Topbaşı NM. The Size and Shape of the Sella Turcica in Adolescent Orthodontic Patients with Different Vertical Growth Paterns. Clin Dent Res. 2017;41(1):3-9.

14. Standerwick RG. Analysis of Average Horizontal and Vertical Mandibular Growth Components Relative to the Horizontal Plane as Determined from Cross-sectional Cepha- lometric Normal Standards. J Dent App. 2014;1(4): 55-60.

15. Silverman FN. Roentgen standards for size of the pituitary fossa from infancy through adolescence. Am J Roentgenol. 1957; 78 (3): 451-460.

16. Yasa, Y., Bayrakdar, I. S., Ocak, A., Duman, S. B., \& Dedeoglu, N. Evaluation of Sella Turcica Shape and Dimensions in Cleft Subjects Using Cone-Beam Computed Tomography. Medical principles and practice: international journal of the Kuwait University, Health Science Centre. 2017;26(3), 280-285.

17. Axelsson S, Storhaug K, Kjaer I. Post-natal size and morphology of the sella turcica. Longitudinal cephalometric standards for Norwegians between 6 and 21 years of age. Eur J Orthod. 2004; 26(6): 597-604.

18. Moffitt AH. Discovery of pathologies by orthodontists on lateral cephalograms. Angle Orthod. 2011; 81(1): 58-63.

19. Tetradis S, Kantor ML. Prevalence of skeletal and dental anomalies and normal variants seen in cephalometric and other radiographs of orthodontic patients. Am J Orthod Dentofacial Orthop. 1999; 116(5): 572-577.

20. Canigur Bavbek N, Dincer M. Dimensions and morphologic variations of sella turcica in type 1 diabetic patients. Am J Orthod Dentofacial Orthop. 2014; 145(2): 179-187.

21. Soaker PC, Nawale S. The comparative study of size of sella turcica in different skeletal types in local population: an in vitro study. Indian J Appl Res. 2014; 4: 160-162.

22. Magat G, Ozcan Sener S. Morphometric analysis of the sella turcica in Turkish individuals with different dentofacial skeletal patterns. Folia Morphol. 2018; 77(3): 543-550. 